\title{
The Effect of Mild Rehydration on Freeze-Dried Dipalmitoylphosphatidylcholine (DPPC) Multilamellar Membranes as Observed by Proton NMR and Sorption Isotherm
}

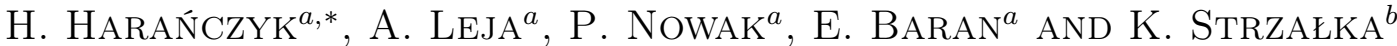

${ }^{a}$ Institute of Physics and ${ }^{b}$ Faculty of Biochemistry, Biophysics and Biotechnology, Jagiellonian University, S. Łojasiewicza 11, 30-348 Cracow, Poland

Between neighbouring bilayers of lyophilized dipalmitoylphosphatidylcholine (DPPC) multilamellar vesicles the total number of water molecules equals $9 \mathrm{H}_{2} \mathrm{O}$ molecules/1 DPPC molecule. One of these molecules is very tightly bound to the lipid molecule, seven are in immobilized (tightly bound) water fraction whereas the last one belongs to mobile water fraction. The rehydration from the gaseous phase of the DPPC model membranes was investigated using hydration kinetics, sorption isotherm, and high power proton relaxometry. The obtained data for DPPC were compared with these obtained for wheat photosynthetic membranes. Rehydrated photosynthetic membranes differ from DPPC model membranes in hydration kinetics. The average hydration time has a similar value: $(22.0 \pm 2.8) \mathrm{h}$ (photosynthetic membrane) and $(19.8 \pm 1.6) \mathrm{h}(\mathrm{DPPC})$, however hydration kinetics was described by one-exponential function for photosynthetic membrane, while for model membrane it shows fine double exponential form. The sigmoidal form of sorption isotherm is better fitted using Dent model than by the Brunauer-Emmett-Teller formula. The Brunauer-Emmett-Teller/Dent deviation parameter $b=0.93$ either for photosynthetic or for model membranes. The mass of water saturating primary water binding sites equals $\Delta M / m_{0}=0.017$ (wheat photosynthetic membranes) and 0.027 (DPPC). The detected by NMR-isotherm study mass of water "sealed" in model membrane structures was about $\Delta M_{\mathrm{s}} / m_{0}=0.182$ (about 7-8 $\mathrm{H}_{2} \mathrm{O}$ molecules $/ 1$ DPPC molecule), and $\Delta M_{\mathrm{s}} / m_{0}=0.066$ for photosynthetic membrane.
\end{abstract}

DOI: $10.12693 /$ APhysPolA.129.179

PACS: 82.56.Na, 87.16.D-

\section{Introduction}

Freeze-drying process may to some extent model the extreme conditions of decreased temperature and of drought stress for plant organellae. It is not clear whether freeze-dried photosynthetic membrane resembles the structure of a native one [1]. However, phosphorus NMR spectra show that after rehydration wheat photosynthetic membrane returns to lamellar phase revealing in the same time the significant contribution of the ${ }^{31} \mathrm{P}-\mathrm{NMR}$ isotropic signal from the small membrane fragments of disrupted membranes [2-4]. Sorption isotherm combined with proton high power relaxation of gradually rehydrated wheat photosynthetic membrane lyophilizates show a presence of "sealed" water fraction, $\Delta M_{\mathrm{s}}$, in dry lyophilizate structures [5], but the heterogeneous composition of photosynthetic membrane lipids hindered the quantified conclusions on the amount of this water fraction, which was estimated by them as $\Delta M_{\mathrm{s}} / m_{0}=0.052$ for native wheat membranes and $\Delta M_{\mathrm{s}} / m_{0}=0.061$ for $1 \mathrm{mM}$ EDTA washed membrane, as expressed in dry mass units, $m_{0}[5]$.

The aim of this paper was to search using hydration kinetics, sorption isotherm and high power proton

*corresponding author; e-mail: hubert.haranczyk@uj.edu.pl relaxometry the presence of "sealed" water fraction in lyophilizates obtained from the lipid preferring lamellar structures. We selected lyophilized DPPC multilamellar vesicles to study their hydration properties because DPPC molecules are often used as lipid model systems for biological membranes.

\section{Materials and methods \\ 2.1. Preparation of lyophilized \\ $D P P C$ multilamellar vesicles}

$950 \mathrm{mg}$ of DPPC (SIGMA) was suspended in $2 \mathrm{ml}$ of chloroform. The obtained suspension was divided for five samples subsequently used in measurements. The supernatant was evaporated, then the sediment was placed under vacuum for $1 \mathrm{~h}$, to remove the remnants of chloroform. Then $5 \mathrm{ml}$ of distilled water was added and every sample was vortexed for $15 \mathrm{~min}$ till the optically uniform milky suspension was obtained (multilamellar vesicles). The obtained preparation was lyophilized overnight at $+4^{\circ} \mathrm{C}$.

\subsection{Rehydration from gaseous phase}

Prior to the hydration courses the lyophilizate was incubated for $75 \mathrm{~h}$ over the surface of $\mathrm{H}_{3} \mathrm{PO}_{4}$ at the relative humidity $p / p_{0}=9 \%$ (see Fig. 1 ), showing mass decrease ca. 0,05 , in units of dry mass.

The hydration time-courses for the DPPC lyophilizates were performed at room temperature $\left(t=22^{\circ} \mathrm{C}\right)$ over 


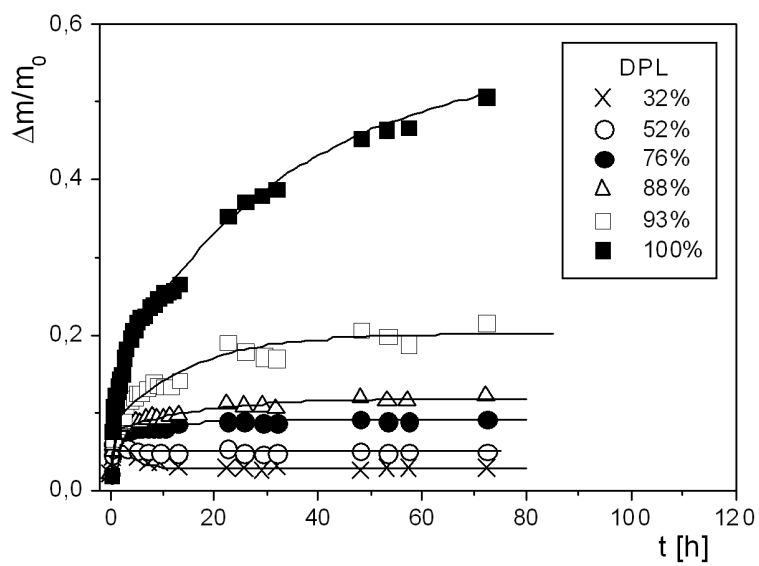

Fig. 1. The rehydration of DPPC multilamellar vesicles lyophilizate performed from gaseous phase at different values of relative humidity, $p / p_{0}$, recorded as relative mass increase, expressed in units of dry mass, $\Delta m / m_{0}$.

the surfaces of saturated solutions of $\mathrm{CaCl}_{2}\left(p / p_{0}=\right.$ $32 \%), \quad \mathrm{Na}_{2} \mathrm{Cr}_{2} \mathrm{O}_{7}\left(p / p_{0}=52 \%\right), \quad \mathrm{Na}_{2} \mathrm{~S}_{2} \mathrm{O}_{3} \quad(76 \%)$, $\mathrm{K}_{2} \mathrm{CrO}_{3}(88 \%), \mathrm{Na}_{2} \mathrm{SO}_{4}(93 \%)$, and over the water surface $\left(p / p_{0}=100 \%\right)$. As a measure of hydration level, the relative mass increase, $\Delta m / m_{0}$, expressed in units of dry mass, was taken.

After completing the hydration courses, the dry mass of the photosynthetic membrane was determined after heating at $60^{\circ} \mathrm{C}$ for $24 \mathrm{~h}$, subsequently weighted, and then for $48 \mathrm{~h}$ at $70^{\circ} \mathrm{C}$. The second course of heating did not cause a significant decrease in the mass of the sample. Higher temperatures were not used to standardize this conditions with the ones applied in dry mass of photosynthetic membrane determination where the temperature above $70^{\circ} \mathrm{C}$ may cause decomposition of some organic constituents of the membrane [6].

\subsection{NMR measurements}

Proton free induction decays (FIDs) were recorded on fully computer controlled spectrometer WNS-65 (Waterloo NMR Spectrometers, St. Agatha, Ontario, Canada) working at the resonance frequency $30 \mathrm{MHz}$. Transmitter power was $400 \mathrm{~W}$; pulse lengths $\pi / 2$ were selected as $1.3 \mu \mathrm{s}$. Data were acquired using Compuscope 2000 card and, then, averaged over 1000 accumulations. Repetition time was $2 \mathrm{~s}$. The measurements were performed at room temperature.

The measured proton FID functions were analysed using one-dimensional data decomposing procedure of the program CracSpin (Jagiellonian University, Cracow, Poland) designed for two-dimensional data decomposition in time domain [7]. In general, due to the properties of the digital oscilloscope card a non-zero constant FID signal component may be taken into account, however its contribution was negligible and for the whole series of measurements was set to zero.

\section{Results \\ 3.1. Hydration kinetics}

The hydration kinetics of DPPC multilamellar vesicles lyophilizate revealed mild dehydration of the air-dry sample placed to $p / p_{0}=9 \%$. In this case the process was described by single exponential function

$$
\Delta m / m_{0}(t)=B^{\mathrm{d}}+A^{\mathrm{d}} \exp \left(-t / t^{\mathrm{d}}\right) .
$$

The hydration of the DPPC lyophilizate from the gaseous phase takes place at the relative humidity, $p / p_{0}$, in the range between $32 \%$ and $100 \%$ (Fig. 1 ). For $p / p_{0}=32 \%$, the saturation hydration level did not differ significantly from the residual humidity of the air-dry lyophilizate, thus, the effects of opening of the desiccator decreased the quality of fitted function, and solely the saturation hydration level value was taken as a result. The hydration kinetics at $p / p_{0}=52 \%$ was less noisy and was successfully fitted by the single exponential function

$$
\Delta m / m_{0}(t)=B^{\mathrm{h}}+A_{1}^{\mathrm{h}}\left[1-\exp \left(-t / t_{1}^{\mathrm{h}}\right)\right],
$$

where $t^{\mathrm{d}}$ is the dehydration time, $B^{\mathrm{d}}$ is the equilibrium hydration at $p / p_{0}=9 \%$, and $A^{\mathrm{d}}$ is the hydration surplus of an air-dry sample. Fitted value of $B^{\mathrm{d}}=2.3 \pm 0.004$.

For $p / p_{0}=76 \%$ and higher the hydration kinetics was fitted well with double exponential function

$$
\Delta m / m_{0}(t)=B^{\mathrm{h}}+A_{1}^{\mathrm{h}}\left[1-\exp \left(-t / t_{1}^{\mathrm{h}}\right)\right],
$$

where $t_{1}^{\mathrm{h}}$ and $t_{2}^{\mathrm{h}}$ are the hydration times of both water fractions recorded, $B^{\mathrm{h}}$ is the saturation hydration of airdry lyophilizate, and $A_{1}^{\mathrm{h}}$ and $A_{2}^{\mathrm{h}}$ are the saturation hydration levels for first and second water fractions, respectively.

\section{TABLE I}

Mild rehydration kinetics of DPPC multilamellar membrane lyophilizates performed from gaseous phase. The $p / p_{0}$ is the relative humidity, the fitted parameters were as defined by Eq. (1) and Eq. (2) (see text). The errors (in parenthesis) are the standard errors of fitted parameters.

\begin{tabular}{c|c|c|c|c|c}
\hline \hline$p / p_{0}[\%]$ & $B^{\mathrm{h}}$ & $A_{1}^{\mathrm{h}}$ & $t_{1}^{\mathrm{h}}[\mathrm{h}]$ & $A_{2}^{\mathrm{h}}$ & $t_{2}^{\mathrm{h}}[\mathrm{h}]$ \\
\hline 32 & \multicolumn{5}{|c}{$0.030(0.001)($ see text) } \\
52 & 0.022 & 0.029 & 0.120 & - & - \\
& $(0.003)$ & $(0.002)$ & $(0.030)$ & & \\
76 & 0.022 & 0.052 & 0.200 & 0.017 & 12.1 \\
& $(0.003)$ & $(0.001)$ & $(0.011)$ & $(0.001)$ & $(1.4)$ \\
88 & 0.023 & 0.052 & 0.319 & 0.044 & 16.9 \\
& $(0.006)$ & $(0.002)$ & $(0.030)$ & $(0.002)$ & $(1.9)$ \\
93 & 0.023 & 0.058 & 0.158 & 0.122 & 14.7 \\
& $(0.019)$ & $(0.009)$ & $(0.062)$ & $(0.005)$ & $(1.9)$ \\
100 & 0.067 & 0.084 & 1.126 & 0.412 & 35.1 \\
& $(0.040)$ & $(0.008)$ & $(0.259)$ & $(0.005)$ & $(1.2)$
\end{tabular}

Table I sets up the values of parameter fitted for hydration kinetics performed at varied relative humidity. The obtained value of minimal hydration level, scaled to that one at $p / p_{0}=9 \%$ and averaged for all hydration courses, except the one at $p / p_{0}=100 \%$, was equal to $B^{\mathrm{h}}=0.023 \pm 0.008$, which is the value close to the 
numbers obtained for lyophilized native photosynthetic membrane $\left(B^{\mathrm{h}}=0.022 \pm 0.005\right)$ [5], suggesting the similar nature of the surfaces investigated.

\subsection{Sorption isotherm}

The saturation hydration level of the sample, $C=B^{\mathrm{d}}$, $C=A^{\mathrm{h}}+B^{\mathrm{h}}, C=A^{\mathrm{h}}+B^{\mathrm{h}}$, for $p / p_{0}=9 \%, p / p_{0}=32 \%$ and $52 \%$, and for higher relative humidities, respectively, was then applied to construction the sorption isotherm, which revealed a sigmoidal form (Fig. 2).

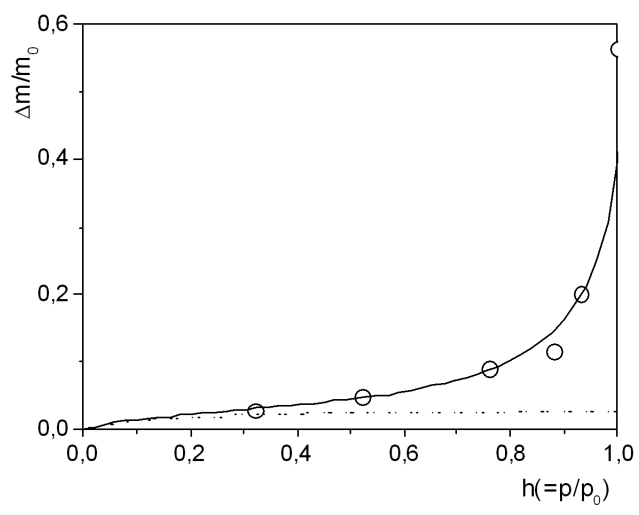

Fig. 2. The sigmoidal in form sorption isotherm recorded at room temperature for the lyophilizate of DPPC multilamellar vesicles. Open circles show experimental data, solid line - total sorption, dotted line shows the population of monolayer sorption, both predicted by Dent isotherm (see text). $h$ is relative humidity expressed in absolute units.

Two models properly describe a sigmoidal form of sorption isotherm, namely BET model (Brunauer-EmmettTeller) (Eq. (4)) [8] and Dent model (Eq. (5)) [9, 10].

$$
\begin{aligned}
& C(h)=\frac{\Delta m}{m_{0}}(h)=\frac{\Delta M}{m_{0}} \frac{b_{1} h}{(1-h)\left(1+b_{1} h-h\right)}, \\
& C(h)=\frac{\Delta m}{m_{0}}(h)=\frac{\Delta M}{m_{0}} \frac{b_{1} h}{(1-b h)\left(1+b_{1} h-b h\right)} .
\end{aligned}
$$

Both these models distinguish two types of water binding sites: (i) "primary" binding sites - directly to the adsorbent surface, and (ii) "secondary" binding sites, usually weaker water binding sites to the already bound water molecules or to the adsorbent surface, but with water less affinity. Although these models were introduced under the assumption that adsorbate molecules arrange in layers, they do not differentiate between second one and next water layers. Both equations are similar in form but they differ in coverage of 2 nd and subsequent water layers. The BET model assumes that the population of $(n+1)$-th layer in units of $n$-th layer

$$
\left.\frac{S_{n+1}}{S_{n}}\right|_{h=1}=b
$$

is equal to $1(b=1)$, whereas Dent considers the decrease of population with the number of bound water layer: $b \geq 1$. The discrepancy of parameter $b$ from unity is a measure of applicability of the Dent model in any given case. Other parameters, which are common for BET and for Dent model are $\Delta M / m_{0}$, the total mass of water saturating primary water binding sites, as expressed in units of dry mass, and $S_{0} /\left.N\right|_{h=1}=1 / b_{1}$, which equals the contribution of empty primary water binding sites at $h=1$. To distinguish of the applicability of both models usually the sorption isotherm is presented in the form

$$
\frac{h}{\Delta m / m_{0}}=A^{\prime}+B^{\prime} h-C^{\prime} h^{2},
$$

where $B^{\prime}=C^{\prime}-A^{\prime}$ for BET model. The sorption parameters may be easily calculated as

$$
\begin{aligned}
& b=\frac{\sqrt{B^{\prime 2}+4 A^{\prime} C^{\prime}}-B^{\prime}}{2 A^{\prime}}, \\
& b_{1}=\frac{B^{\prime}}{A^{\prime}}+2 b, \\
& \Delta M / m_{0}=\frac{1}{A^{\prime} b_{1}}
\end{aligned}
$$

for the Dent model

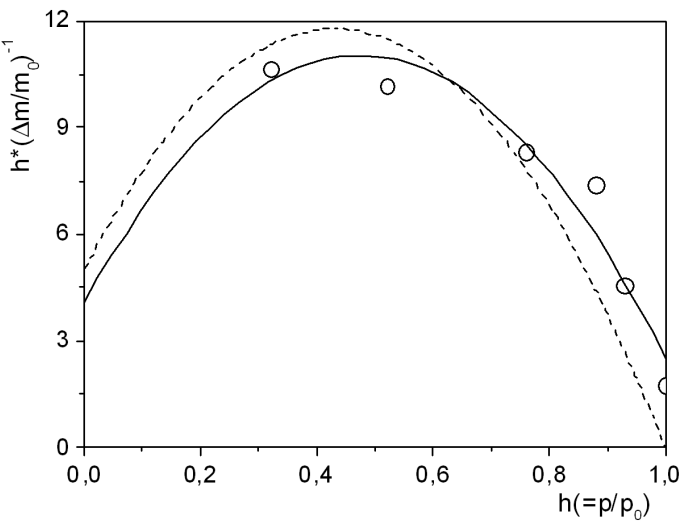

Fig. 3. The sorption isotherm for lyophilizate of DPPC multilamellar vesicles expressed in terms of $\frac{h}{\Delta m / m_{0}}$ shown versus $h$. The experimental data (open circles), BET isotherm (dotted line) and Dent isotherm (solid line).

Figure 3 shows the BET and Dent models applied to the sorption data for DPPC multilamellar vesicles lyophilizate. The Dent model substantially better fits sorption data for rehydrated lyophilizate of DPPC multilamellar vesicles (coefficient of determination, $R^{2}$, is much closer to 1 for the Dent model than for the BET model). The parameters of the Dent isotherm in parabolic form (Eq. (7)) were equal to $A^{\prime}=4.1 \pm 4.1$, and $B^{\prime}=29.1 \pm$ 13.7 , and $C^{\prime}=30.7 \pm 10.3$. The mass of water saturating primary water binding sites equals $\Delta M / m_{0}=0.0272$, which is the value slightly higher than for native photosynthetic membrane lyophilizate (0.024) and for the photosynthetic membrane lyophilizate washed from paramagnetic ions by $1 \mathrm{mM}$ EDTA (0.017) [5]. The measure of population of subsequent secondary bound water layers equals $b=0.931$, which is similar to $1 \mathrm{mM}$ EDTAwashed photosynthetic membranes (0.929) and to native photosynthetic membrane (0.896) [5]. The fraction of unbound primary water binding sites at $h=1$ is equal to 
$1 / b_{1}=11.2 \%$, which is in between the numbers for native photosynthetic membrane lyophilizates $(2.2 \%)$ and for $1 \mathrm{mM}$ EDTA-washed ones (14.6\%) [5].

\subsection{Proton magnetic relaxation}

Proton FID recorded for rehydrated from gaseous phase lyophilizates of DPPC multilamellar vesicles (see Fig. 4) is well fitted by the superposition of one Gaussian function and two exponential functions

$$
\begin{aligned}
& \operatorname{FID}(t)=S_{0} \exp \left(-\left(t / T_{G, 0}^{*}\right)^{2}\right) \\
& \quad+L_{1} \exp \left(-t / T_{2,1}^{*}\right)+L_{2} \exp \left(-t / T_{2,2}^{*}\right),
\end{aligned}
$$

where $S_{0}$ is the amplitude of the Gaussian component, $T_{G, 0}^{*}$ is the 1/e-decay time constant for Gaussian decay; $L_{1}$ and $L_{2}$ are the amplitudes of the exponential components, $T_{2,1}^{*}$ and $T_{2,2}^{*}$ are the relaxation time constants for both exponential components, respectively. We used the 1 /e-value instead of the one-half value for solid Gaussian curve to allow a direct comparison between the characteristic time constant used to describe the solid and the ones describing the liquid signal components [11-13].

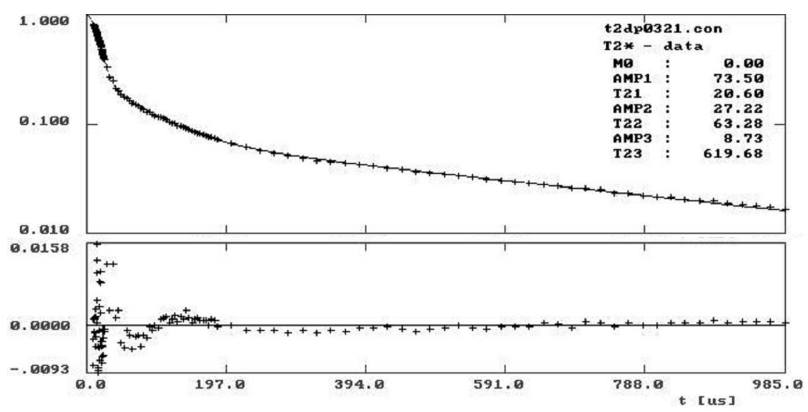

Fig. 4. Proton FID recorded for lyophilizate of DPPC membrane rehydrated from $p / p_{0}=45 \%$ at room temperature; pulse length $\pi / 2=1.3 \mu \mathrm{s}, x$-axis: time in $\mu \mathrm{s}$, $y$-axis: signal amplitude measured in arbitral units. Figure is a typical printout from one-dimensional fitting procedure of CracSpin. Upper plot shows FID, whereas the lower one presents the residual function [7].

\subsection{Hydration dependence of proton FID}

Figure 5 shows the hydration dependence of the proton FID times for the resolved spin subsystems of protons in rehydrated from gaseous phase lyophilizates of DPPC multilamellar vesicles. The time constant for the shortest Gaussian weakly if at least depends on hydration level of the sample with the value $T_{G .0}^{*} \approx 20 \mu \mathrm{s}$, which is close to the value for other biological solid porous matrices, e.g. for bark and bast [14], of lichen thallus [11$13]$, of dentine and in dental enamel $[15,16]$ and of the wheat seed [4]. Thus, we assigned the Gaussian component, $S_{0}$, to the solid matrix of DPPC multilamellar vesicle lyophilizate. As this component does not vary much with hydration level, we used the amplitude of this component as a unit to measure the amplitudes of the other signal components.

Figure 6 shows the hydration dependence of the amplitudes of both liquid signal components $L_{1}$ and $L_{2}$

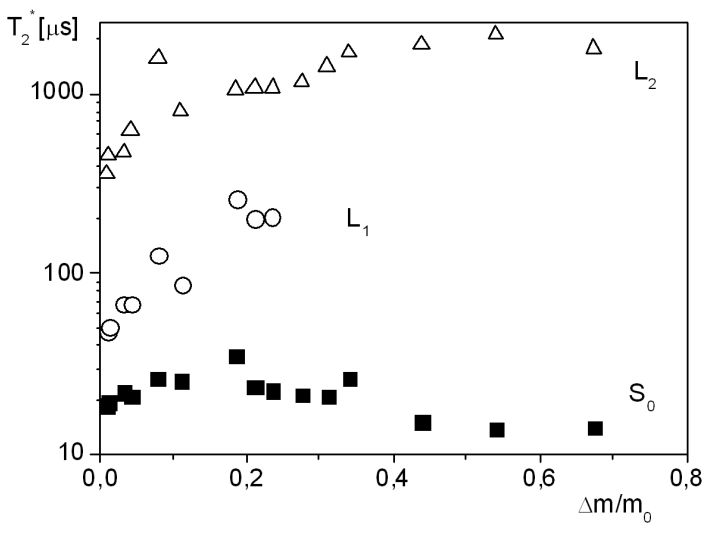

Fig. 5. Proton spin-spin relaxation times calculated from FID for rehydrated lyophilizate of DPPC multilamellar vesicles as a function of hydration level, $\Delta m / m_{0}$, expressed in units of dry weight: solid squares - proton solid component (Gaussian), $S_{0}$, open circles - tightly bound water (exponential component), $L_{1}$, and open triangles - loosely bound water (exponential), $L_{2}$.

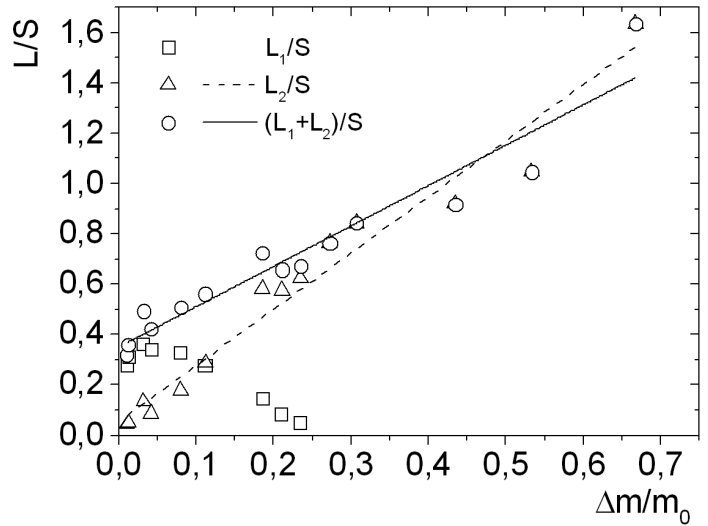

Fig. 6. The amplitudes of FID liquid components expressed in units of solid signal, $S_{0}$, recorded for rehydrated lyophilizates of DPPC multilamellar vesicles as a function of hydration level, $\Delta m / m_{0}$, expressed in units of dry weight: open squares $-L_{1}$ component; open triangles - $L_{2}$ component, and dashed line - fitted linear function; open circles - the total liquid signal $\left(L_{1}+L_{2}\right)$, and solid line - fitted linear function, (see text).

for rehydrated lyophilizate of DPPC multilamellar vesicles. The amplitude of liquid, $L_{2}$, component linearly depends on hydration level, according to the equation: $L_{2} / S=a \Delta m / m_{0}$, for $a=2.31 \pm 0.09$; the value of spinspin relaxation time is about $T_{2,2}^{*} \approx 1 \mathrm{~ms}$ suggesting that $L_{2}$ component comes from loosely bound water fraction. As the longest $T_{2}^{*}$ components are limited in FID measurement by the $B_{0}$-field non-uniformities, the $L_{2}$ component may be the average of several loosely bound and free water fractions being in fast exchange regime [17]. Due to numerical reasons (increasing contribution of $L_{2}$ ) the magnitude of liquid $L_{1}$ component was not resolved with the accuracy sufficient to present consistent dependence on hydration level, although the value of $T_{2,1}^{*} \approx 100 \mu \mathrm{s}$ suggests that $L_{1}$ component comes from water tightly 
bound on the membrane surface. Such an attribution of both liquid components is consistent with the proton relaxation data for water bound to photosynthetic membranes [5], for lichen thalli [11-13], dentine and dental enamel [15, 16], bark and bast [14], and water bound in inorganic model system of controlled porous glass [18].

The hydration dependences of total liquid signal $\left(L_{1}+\right.$ $\left.L_{2}\right) / S_{0}$ in units of solid (see Fig. 6) is well fitted by the linear function $\left(L_{1}+L_{2}\right) / S_{0}=A+k \Delta m / m_{0}$ with $A=$ $0.36 \pm 0.03$, and $k=1.54 \pm 0.11$.

\subsection{NMR sorption isotherm}

The total proton signal of liquid component, $\left(L_{1}+\right.$ $\left.L_{2}\right) / S_{0}$, coming from water bound to the surfaces of rehydrated lyophilizate of DPPC multilamellar vesicles was used to construct the NMR-isotherm (Fig. 7). The fit pa-

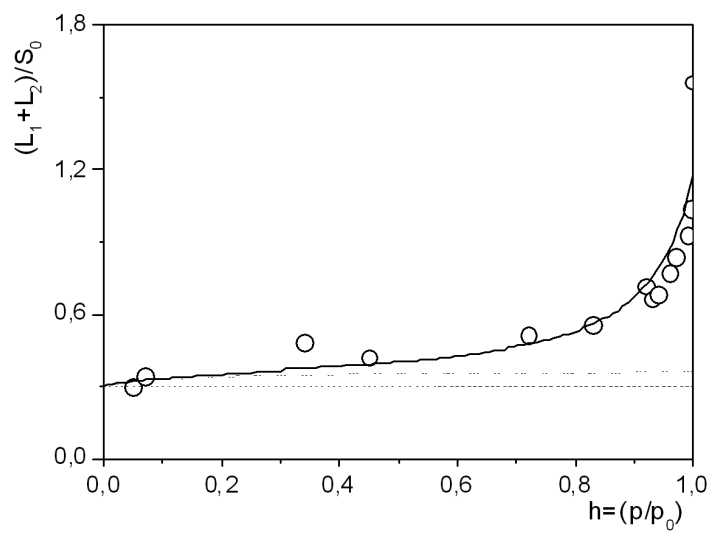

Fig. 7. The sorption isotherm for rehydrated from lyophilizate DPPC multilamellar vesicles as a function of the total liquid NMR signal expressed in units of solid component, $\left(L_{1}+L_{2}\right) / S_{0}$. Solid line - the sorption isotherm with the parameters obtained from fitting of gravimetric data, and then linearly fitted to the NMR data, with the parameters: $L_{1,0} / S_{0}$ - the contribution of "sealed" water, and $k$ - proportionality coefficient between amplitude of the liquid NMR signal and the mass of the water added. Dotted line - shows the level of the "sealed" water, $L_{1,0} / S_{0}$, whereas dashed line the level of primary binding site population.

rameters were the amplitude of water signal coming from the sealed water pool, $L_{1,0} / S_{0}$, and the proportionality coefficient, $k$, which is the slope of the NMR signal amplitude hydration dependence. The minimized function was

$$
\begin{aligned}
& \frac{S_{1}+L_{1}+L_{2}}{S_{0}}(h)= \\
& \frac{L_{1,0}}{S_{0}}+k \frac{\Delta M}{m_{0}} \frac{b_{1} h}{(1-b h)\left(1+b_{1} h-b h\right)},
\end{aligned}
$$

where the values $\Delta M / m_{0}, b$ and $b_{1}$ were not fitted but taken from fits of sorption isotherm.

So obtained sorption isotherm fits the NMR data sufficiently well, with the parameters $L_{1,0} / S_{0}=0.30 \pm 0.06$ and $k=2.23 \pm 0.28$. The obtained values do not much differ from those obtained from the analysis of $L_{2} / S_{0}$ dependence, and from those obtained from the analy-
TABLE II

The proportionality coefficient $k$ calculated for different methods.

\begin{tabular}{c|c|c}
\hline \hline & coefficient $k$ & $L_{1,0} / S_{0}$ \\
\hline $\begin{array}{c}\text { the slope of } L_{2} / S_{0} \\
\text { hydration dependence }\end{array}$ & $2.31 \pm 0.09$ & \\
the slope of total $\left(L_{1}+L_{2}\right) / S_{0}$ & $1.54 \pm 0.11$ & $0.36 \pm 0.03$ \\
$\begin{array}{c}\text { hydration dependence } \\
\text { NMR sorption isotherm }\end{array}$ & $2.23 \pm 0.28$ & $0.30 \pm 0.06$
\end{tabular}

sis of the total liquid signal dependence, $\left(L_{1}+L_{2}\right) / S$ (see Table II).

\section{Conclusions}

In proton FID recorded for rehydrated lyophilizates of native wheat photosynthetic membrane the second Gaussian component, $S_{1}$, with the magnitude depending on hydration level of the sample was observed, which comes from the highly immobilized water fraction in the vicinity of paramagnetic ions on the membrane surface [5]. In DPPC lyophilized membranes such a component was not detected, which confirms their interpretation.

(i) Rehydrated photosynthetic membranes differ from DPPC model membranes in hydration kinetics. Although the hydration time has a similar value: $(22.0 \pm$ $2.8) \mathrm{h}$ and $(19.8 \pm 1.6) \mathrm{h}$ (major long component), respectively, the form of the fitted function is single (averaged by numerical procedure from the components coming from the several membrane components) exponential for photosynthetic membrane, while for model membrane it shows fine double exponential form.

(ii) The sigmoidal form of sorption isotherm is better fitted using Dent model than by BET formula. The BET/Dent deviation parameter $b=0.93$ either for photosynthetic or for model membranes. The mass of water saturating primary water binding sites equals $\Delta M / m_{0}=0.17$ and 0.027 , for photosynthetic and for model membrane, respectively.

(iii) Proton FID is well fitted by a superposition of solid Gaussian and one or two exponential components coming from water bound in the system. As a photosynthetic membrane was washed out from the paramagnetic ions (by $1 \mathrm{mM}$ EDTA), no Gaussian component coming from highly immobilized water molecules was recorded.

(iv) The detected by NMR-isotherm study mass of water "sealed" in model membrane structures was about $\Delta M_{\mathrm{s}} / m_{0}=0.182$ (about $7-8 \mathrm{H}_{2} \mathrm{O}$ molecules $/ 1$ DPPC molecule), and $\Delta M_{\mathrm{s}} / m_{0}=0.066$ for photosynthetic membrane. The difference suggests that in photosynthetic membrane lyophilizate water is "sealed" mainly in lipid structures (ca. $48 \%$ of membrane mass), moreover the hexagonal phase tubulae formed by photosynthetic membrane lipids may cause further reduction in "sealed" water mass.

(v) The estimated from the $L / S$ hydration dependence contribution of "sealed" water fraction shows the number of $9 \mathrm{H}_{2} \mathrm{O}$ molecules/1 DPPC molecule. This value equals 
such a number recorded for DPPC bilayer in liquid crystalline phase [19], and is within the number of tightly bound water molecules plus part of main hydration shell for hydrated DPPC bilayer [20].

(vi) The estimated number of molecules of water immobilized fraction calculated from the $L / S$ hydration dependence is equal to $8 \mathrm{H}_{2} \mathrm{O}$ molecules/1 DPPC molecule, which means that in lyophilized multilamellar DPPC vesicles the number of water trapped between bilayers is sufficient to contain one molecule of mobile (loosely bound) water fraction.

(vii) Summarizing, this experiments show that between bilayers of lyophilized DPPC multilamellar vesicles the total number of water molecules equals $9 \mathrm{H}_{2} \mathrm{O}$ molecules/1 DPPC molecule. One of these molecules is very tightly bound to the lipid molecule, seven of them belong to immobilized (tightly bound) water fraction whereas the last one comes from mobile water fraction.

\section{Acknowledgments}

The research was carried out with the equipment purchased thanks to the financial support of the European Regional Development Fund in the framework of the Polish Innovation Economy Operational Program (contract no. POIG.02.01.00-12-023/08), and also financed by the Polish Ministry of Science and Higher Education (MNiSW, contract no. 7150/E-338/M/2015). Faculty of Biochemistry, Biophysics and Biotechnology of Jagiellonian University is a partner of the Leading National Research Center (KNOW) supported by the Ministry of Science and Higher Education.

\section{References}

[1] D. Latowski, J. Kruk, K. Burda, M. SkrzyneckaJaskier, A. Kostecka-Gugała, K. Strzałka, Eur. J. Biochem. 269, 4656 (2002).

[2] H. Harańczyk, K. Strzałka, T. Bayerl, G. Klose, J.S. Blicharski, in: Mat. XVI Ogólnopol. Sem. MRJ, Kraków 1983, Report IFJ No. 1237/PL, 1984, p. 43 .
[3] H. Harańczyk, K. Strzałka, T. Bayerl, G. Klose, J.S. Blicharski, Photosynthetica 19, 414 (1985).

[4] H. Harańczyk, K. Strzałka, W. Dietrich, J.S. Blicharski, J. Biol. Phys. 21, 125 (1995).

[5] H. Harańczyk, A. Leja, K. Strzałka, Acta Phys. Pol. A 109, 389 (2006).

[6] D.F. Gaff, Oecologia (Berl.) 31, 95 (1977).

[7] W. Węglarz, H. Harańczyk, J. Phys. D Appl. Phys. 33, 1909 (2000).

[8] S. Brunauer, P.H. Emmett, E. Teller, J. Am. Chem. Soc. 60, 309 (1938).

[9] R.W. Dent, Textile Res. J. 47, 145 (1977).

[10] A.J. Hailwood, S. Horrobin, Trans. Farad. Soc. 42B, 84 (1946).

[11] H. Harańczyk, S. Gaździński, M.A. Olech, New Phytologist 138, 191 (1998).

[12] H. Harańczyk, S. Gaździński, M. Olech, in: New Aspects in Cryptogamic Research, Contribution in Honour of Ludger Kappen. Bibl. Lichenol., Vol. 75, 2000, p. 265.

[13] H. Harańczyk, A. Pietrzyk, A. Leja, K. Strzałka, Acta Phys. Pol. A 109, 411 (2006).

[14] H. Harańczyk, W.P. Węglarz, S. Sojka, Holzforschung 53, 299 (1999).

[15] N. Funduk, D.W. Kydon, L.J. Schreiner, H. Peemoeller, L. Miljković, M.M. Pintar, Magn. Res. Med. 1, 66 (1984).

[16] N. Funduk, G. Lahajnar, L. Miljković, S. Skocajić, D.W. Kydon, L.J. Schreiner, M.M. Pintar, Zobozdrav. Vestn. 41, 139 (1986).

[17] J.R. Zimmerman, W.E. Brittin, J. Phys. Chem. 61, 1328 (1957)

[18] H. Harańczyk, K.G. Soga, R.J. Rumm, M.M. Pintar, Magn. Res. Imag. 9, 723 (1991).

[19] G. Nimtz, Phys. Scr. T13, 172 (1986).

[20] G. Klose, K. Gawrisch, Stud. Biophys. 84, 21 (1981). 